CSF Glucose was documented in $80 \%$ of cases and levels were low $(<2.5 \mathrm{mmol} / \mathrm{L})$ in $47 \%$. TB PCR was performed on 15 samples (38\%), 2 (13\%) were positive. Five CSF samples were not sent for AFB or culture. No samples were smear positive, 26\% of CSF samples were culture positive; one was Isoniazid resistant.

7 patients died (one death attributed to TB chemotherapy), 3 became fully dependent for all activities of daily living and 6 patients had significant cognitive or neurological deficit.

Conclusions CNS TB causes significant morbidity and mortality. CSF examination should always be performed if feasible. Imaging by MRI should be considered in all patients with suspected TB meningitis in view of the much higher diagnostic yield compared to CT.

\section{P260 POTENTIAL IMPACT OF THE 2015 NICE CONSULTATION GUIDELINE FOR TUBERCULOSIS ON THE NUMBER OF CHILDREN ASSESSED AND TREATED FOR TB INFECTION AND DISEASE IN THE UK}

L Turnbull, C Bell, F Child. Central Manchester University Hospitals NHS Foundation Trust, Manchester, UK

\subsection{6/thoraxinl-2015-207770.396}

Background NICE Tuberculosis (TB) guideline 2015 recommends all children, regardless of BCG status, with Mantoux $\geq 5$ $\mathrm{mm}$ induration receive treatment for latent $\mathrm{TB}$ once active TB has been excluded. The 2011 version defines a positive Mantoux as $\geq 6 \mathrm{~mm}$ (no prior $\mathrm{BCG}$ ) and $\geq 15 \mathrm{~mm}$ (prior BCG). NICE 2011 recommends screening of household contacts of all cases of TB compared with the 2015 guideline which recommends screening of contacts of pulmonary TB only.

Objectives To establish the impact of the change in NICE recommendations on the number of children assessed and treated for latent TB infection (LTBI) or TB disease in our department.

Methods We performed a retrospective analysis of all children.

Results 445 patients were referred, 75 with symptoms, 138 new entrants, 63 non-pulmonary contacts and 169 pulmonary contacts.

Of those with symptoms, 5 had positive Mantoux (NICE 2011) compared with 18 (NICE 2015). In this group 0/75 were treated for LTBI and 7/75 for TB disease.

Results of patients referred for contact tracing/new entrant screening are shown in Table 1 . Two contacts with LTBI and 1 with TB disease (all IGRA positive) would have been missed by the 2011 guideline but identified in 2015 .

Abstract P260 Table 1 Number of patients referred for contact tracing or new entrant screening by Mantoux test result and TB disease status

\begin{tabular}{llllll}
\hline Guideline & Mantoux & LTBI & TB disease & No LTBI or TB disease & Total \\
\hline 2011 & Positive & 9 & 3 & 2 & 14 \\
& Negative & 3 & 1 & 352 & 356 \\
$2015^{*}$ & Positive & 11 & 4 & 32 & 47 \\
& Negative & 1 & 0 & 259 & 260 \\
\hline
\end{tabular}

*Non-pulmonary contacts not included in 2015 data.
Following NICE 201563 non-pulmonary contacts would not have been seen. None of these had LTBI or TB disease. Of the remaining 307 contacts/new entrants $47(15 \%)$ had a positive Mantoux of whom 11(4\%) had LTBI and 4(1\%) TB disease. Conclusion $37 \%$ more children will be investigated and treated for TB infection/disease under the new NICE TB guideline. In a 12 month period in our clinic this represents 33 additional children with 1 extra case of TB disease and 2 cases of LTBI identified.

\section{P261 CHEMOPROPHYLAXIS FOR LTBI FOLLOWING MASS SCREENING IN THE WORKPLACE: UNEXPECTED OUTCOMES IN THE OVER 35S}

${ }^{1} Y$ Abunga, ${ }^{1} \mathrm{M}$ Day, ${ }^{2} \mathrm{~J}$ Williams, ${ }^{3} \mathrm{JP}$ Mamo, ${ }^{1} \mathrm{SO}$ Brij. ${ }^{1}$ Peterborough City Hospital, Peterborough, UK; ${ }^{2}$ Cambridge and Peterborough Foundation Trust, Peterborough, UK; ${ }^{3}$ Western Community Hospital, Southampton, UK

\subsection{6/thoraxjnl-2015-207770.397}

Introduction In 2014, over 500 workers in a local factory were screened for TB. 3 cases of active pulmonary TB were identified and seen in the next weekly TB Clinic. 128 workers were identified for further assessment by the local TB Service, of whom 100 were found to be IGRA-reactive. This was declared a major incident and a TB Action Group was set up to facilitate additional out-of-hours TB clinics.

Methods The local CCG commissioned the additional TB clinics at standard respiratory out-patient tariff: approximately 35 workers were to be assessed by 5 TB clinicians in 2 weekly sessions (18:00-21:00 - 20 min slots) for the first 2 weeks, so that by week 3, all workers would be assessed. As in the weekly TB Clinic, the TB Pharmacy Team would be present to dispense TB medication with drug information leaflets and contact details. Chemoprophylaxis for LTBI was offered to all workers with reactive IGRA and no evidence of active TB independent of their age despite NICE guidance.

Results Of the 100 workers with reactive IGRA: 18 did not attend; 82 were offered chemoprophylaxis of whom 15 declined treatment; 67 started chemoprophylaxis of whom only 33 completed 3 months treatment with rifampicin and isoniazid. The rate of completion of chemoprophylaxis in the eligible group was $9 / 35(25.7 \%)$ compared to $24 / 47(51.1 \%)$ in the over 35 year olds. There was a transient rise in liver enzymes in 1 worker aged over 35 but otherwise there were no other significant side-effects.

Discussion It is difficult to deny chemoprophylaxis for LTBI infection on the basis of age in a large screening event such as this when the average age is 40 (range 17-63) and the oldest member of the cohort tolerated chemoprophylaxis without significant side-effects. The reasons for reluctance to continue chemoprophylaxis in this cohort are poorly understood although lifestyle issues such as reducing alcohol consumption were perceived to be barriers to successful completion of treatment.

Conclusion Chemoprophylaxis for LTBI in this cohort was not tolerated in the eligible population. When undertaking mass screening, it is important to ensure that non-standard treatment is funded, if this is to be offered. Treatment of the over 35s significantly increased the workload and cost of this cohort, although uptake of chemoprophylaxis and successful completion was twice that of the workers aged 35 or less. 